\title{
Mining massive genomic data of two Swiss Braunvieh cattle populations reveals six novel candidate variants that impair reproductive
} success

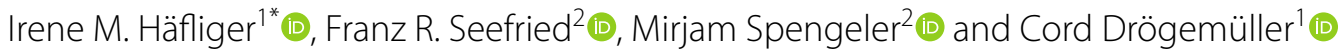

\begin{abstract}
Background: This study was carried out on the two Braunvieh populations reared in Switzerland, the dairy Brown Swiss (BS) and the dual-purpose Original Braunvieh (OB). We performed a genome-wide analysis of array data of trios (sire, dam, and offspring) from the routine genomic selection to identify candidate regions showing missing homozygosity and phenotypic associations with five fertility, ten birth, and nine growth-related traits. In addition, genomewide single SNP regression studies based on 114,890 single nucleotide polymorphisms (SNPS) for each of the two populations were performed. Furthermore, whole-genome sequencing data of 430 cattle including 70 putative haplotype carriers were mined to identify potential candidate variants that were validated by genotyping the current population using a custom array.

Results: Using a trio-based approach, we identified 38 haplotype regions for BS and five for OB that segregated at low to moderate frequencies. For the BS population, we confirmed two known haplotypes, BH1 and BH2. Twenty-four variants that potentially explained the missing homozygosity and associated traits were detected, in addition to the previously reported TUBD1:p.His210Arg variant associated with BH2. For example, for BS we identified a stop-gain variant (p.Arg57*) in the MRPL55 gene in the haplotype region on chromosome 7. This region is associated with the 'interval between first and last insemination' trait in our data, and the MRPL55 gene is known to be associated with early pregnancy loss in mice. In addition, we discuss candidate missense variants in the CPT1C, MARS2, and ACSL5 genes for haplotypes mapped in BS. In OB, we highlight a haplotype region on chromosome 19, which is potentially caused by a frameshift variant (p.Lys828fs) in the LIG3 gene, which is reported to be associated with early embryonic lethality in mice. Furthermore, we propose another potential causal missense variant in the TUBGCP5 gene for a haplotype mapped in OB.

Conclusions: We describe, for the first time, several haplotype regions that segregate at low to moderate frequencies and provide evidence of causality by trait associations in the two populations of Swiss Braunvieh. We propose a list of six protein-changing variants as potentially causing missing homozygosity. These variants need to be functionally validated and incorporated in the breeding program.
\end{abstract}

*Correspondence: irene.haefliger@vetsuisse.unibe.ch ${ }^{1}$ Institute of Genetics, Vetsuisse Faculty, University of Bern, Bremgartenstrasse 109a, 3001 Bern, Switzerland

Full list of author information is available at the end of the article

\section{Background}

Good female fertility in cattle is of high interest in agriculture, to maintain production and genetic diversity within a population [1]. Therefore, traits that affect growth, birth, and fertility are important to breeders original author(s) and the source, provide a link to the Creative Commons licence, and indicate if changes were made. The images or other third party material in this article are included in the article's Creative Commons licence, unless indicated otherwise in a credit line to the material. If material is not included in the article's Creative Commons licence and your intended use is not permitted by statutory regulation or exceeds the permitted use, you will need to obtain permission directly from the copyright holder. To view a copy of this licence, visit http://creativecommons.org/licenses/by/4.0/. The Creative Commons Public Domain Dedication waiver (http://creativeco mmons.org/publicdomain/zero/1.0/) applies to the data made available in this article, unless otherwise stated in a credit line to the data. 
and semen suppliers [1]. In the current Swiss breeding programs, the traits used or intended for breeding value estimation are e.g., non-return rate, the time between calving, birth weight, calving ease, multiple birthing, rearing success, traits regarding survival within a defined period, and slaughter weight. Several studies have shown that fertility ability in dairy cattle has declined in international breeds [2, 3], the main reason being the unfavourable correlation between milk production and fertility [2-4]. This has led to increased efforts to include and prioritize fertility traits in breeding schemes and to identify the genetic causes in female reproduction, in order to breed more fertile cows. Generally, these traits have a low heritability, however, with the improvement of genomic methods, including the implementation of genomic selection (GS) [5], the reliability of breeding values can be increased [6]. Genomic selection is based on single nucleotide polymorphism (SNP) array genotyping and is especially interesting for highly complex traits with a low to moderate heritability, since it can partially account for Mendelian sampling [5, 6]. Combining comprehensive genotyping of breeding animals and recording of the phenotypes within a breeding program provides a promising database for genetic evaluations, including genome-wide association studies (GWAS). These can be carried out for all recorded phenotypes included in a breeding scheme to identify the underlying quantitative trait loci (QTL). Furthermore, the genetic variation of the population is well represented within the routinely genotyped cattle population. Studies on the search for haplotypes that show a significant reduction in homozygosity have been carried out in many species and breeds (e.g. [7-27]). Haplotypes that show no homozygous carriers may indicate the presence of recessive lethal variants within these regions. In this way, many causative variants have been detected by mining these haplotypes and using whole-genome sequencing data (e.g. [9-18, 26-32]). In the international Brown Swiss population, two haplotype regions have been repeatedly mapped, namely Brown Swiss Haplotype 1 (BH1) on chromosome 7 and Brown Swiss Haplotype 2 (BH2) on chromosome 19 [11, 19]. For $\mathrm{BH} 2$, which is associated with high juvenile mortality, a likely causative variant was found in the TUBD1 gene $[11,19]$. Likewise, various embryonic lethal protein-changing variants have been reported to be associated with haplotypes showing missing homozygosity in other cattle populations [10-18, 27, 33]. These examples demonstrate that short-read-based wholegenome sequencing (WGS) data make it possible not only to identify genomic regions, but also to decipher the entire genetic code of an individual in a time- and cost-efficient manner. Thereby the likely causative variants for monogenic traits segregating in livestock populations can be discovered and systematically selected against [23].

In Switzerland, Original Braunvieh (OB) and Brown Swiss (BS) are agronomically important dual purpose and dairy cattle populations, respectively. Historically, the $\mathrm{OB}$ population is the autochthonous brown Swiss cow with no BS influence and is adapted to the Swiss climate, whereas the modern BS originates from the OB by introgression of North American BS cattle [34]. It has been shown that the two populations segregate genetically from each other and are thus considered independent populations [35]. Both populations occur predominantly in the eastern parts of Switzerland and still have an important role in traditional alpine farming, which is frequently practiced.

The aims of this study were to identify haplotypes associated with growth, birth and fertility traits in two local Braunvieh cattle populations. Based on missing homozygosity screens that focus on trios, linkage disequilibrium analyses, and trait associations, we detected both known and novel haplotype regions segregating at low to moderate frequencies with a negative influence on female reproduction and calf survival. Based on WGS data, we identified potential protein-coding candidate causative variants for selection, including those that impair early embryonic and pre-weaning survival.

\section{Methods}

To give a comprehensive overview of the methods applied, the workflow used to mine the genomic data from SNP arrays, WGS data, and a broad variety of phenotypes for the Swiss BS and OB populations is summarized in Fig. 1.

\section{SNP array data}

The SNP array data used in this study were provided by the breeding associations of the $\mathrm{BS}$ and $\mathrm{OB}$ populations. The cleaned SNP dataset comprised 114,890 SNPs and represents a combination of different SNP arrays. As of 2018, the positions of SNPs were updated to the latest cattle reference sequence ARS-UCD1.2 [36, 37]. Data were imputed by using the software Fimpute v2.2 [38] to correct for erroneously called SNPs and to complete the SNP set for individuals genotyped with a lower chip density. Data were phased by using the Beagle v5.0 software package [39].

The OB dataset included 10,085 genotyped animals, from which 3287 trios (trio: sire, dam, and offspring) and 4360 paternal half-sib groups (pgp: parent - grand-parent; sire, maternal grandsire, and offspring) were derived for the analyses (Table 1). The BS dataset included 48,807 


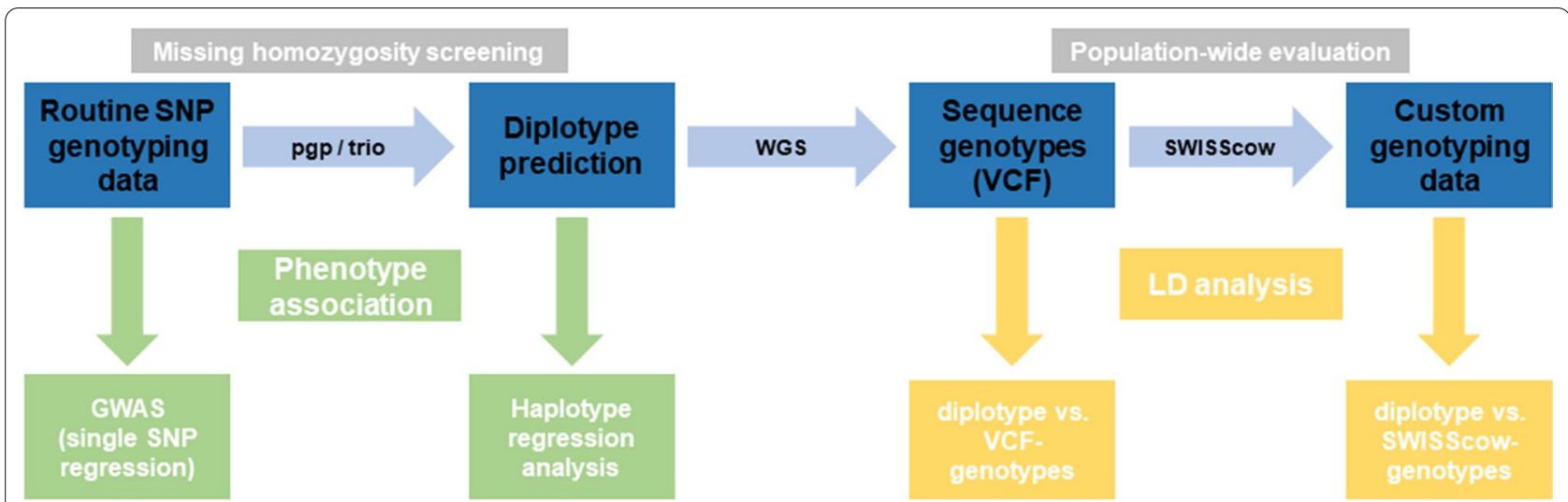

Fig. 1 Workflow of the reverse genetic analyses. Note that the steps of the main genomic analyses that are described in the Methods section are indicated in blue. Further evaluations are shown in green (phenotypic analysis) and yellow (linkage disequilibrium (LD) analysis)

Table 1 Number of genotyped animals from the two Braunvieh cattle populations used for missing homozygosity scan, haplotype, and GWAS analyses

\begin{tabular}{lll}
\hline & $\begin{array}{l}\text { Original } \\
\text { Braunvieh } \\
\text { (OB) }\end{array}$ & Brown Swiss (BS) \\
\hline Number routine genotyped animals & 10,085 & 48,807 \\
$\begin{array}{l}\text { Number trios (sire, dam and offspring) } \\
\text { Number paternal half sib groups- }\end{array}$ & 3287 & 14,450 \\
$\begin{array}{l}\text { pgp (sire, maternal grandsire and } \\
\text { offspring) }\end{array}$ & 4360 & 32,319 \\
\hline
\end{tabular}

genotyped animals, with 14,450 trios and 32,319 half-sib groups (Table 1). The SNP array data were quality filtered by retaining SNPs with a MAF higher than 0.01 and a call rate greater than 0.9 , and individuals with a call rate greater than 0.8 .

\section{Haplotype analysis}

We used the snp1101 software to screen the genomic data for haplotypes that showed a significant deviation from the Hardy-Weinberg equilibrium (HWE) based on the exact test of HWE [40, 41]. The screen was performed for each population and repeated for both the trio-based and the pgp-based approaches. The dataset used for screening was generated by including the genotypes of the Swiss animals born after 2009 and their ancestors. The snp1101 software screens each autosome based on a sliding-window approach, for which we defined a window size of 50 SNPs that represents genome regions ranging from 0.26 to $3.2 \mathrm{Mb}$ with an average length of $1.1 \mathrm{Mb}$. The output is a list of all the significant haplotypes with the number of observed and expected homozygous carriers, the allele frequency and the haplotypes themselves. We carefully defined all the regions of interest as the regions that show a reduction in homozygosity and a significant deviation from HWE with their adjusted p-value for a false discovery rate (FDR) corrected according to the BenjaminiYekutieli procedure [42]. For each region, we chose the most significant haplotype to reduce the number of false positive carriers. We defined the diplotype status for each haplotype of all the animals in the population to select the most relevant animals for whole-genome sequencing. By sequencing healthy breeding animals that are either heterozygous or homozygous carriers of the diplotypes, we increased the probability of sequencing animals that carry the associated causal variants.

\section{Association studies}

Based on the predicted diplotype status, haplotype association studies were carried out for a variety of traits and for each of the observed haplotypes. For the association analyses, routinely available conventional, non-genomic breeding values related to fertility, birth, and growth were used. The individual traits are described in Table 2. Only breeding values with a reliability higher than 0.5 were deregressed according to Garrick et al. [43] and used in a linear mixed model using the genome-wide complex trait analysis (GCTA) software [44]. Thereby, only the animals that have own performance or progeny performances were included in the analysis. The model is as follows: $y_{E B V}=\mu+G+\mathbf{H} \boldsymbol{\beta}+\epsilon$, where $y_{E B V}$ is the deregressed breeding value ( $\mathrm{drEBV}), \mu$ is the average of the deregressed breeding values, $G$ is a random genetic effect based on a genomic relationship matrix (GRM) estimated by the GCTA software [44], $\mathbf{H}$ is a vector of the diplotype status per animal, $\boldsymbol{\beta}$ is a vector of the estimated fixed effects of each haplotype and $\epsilon$ accounts for random variation.

We also performed genome-wide association studies (GWAS) on the same genomic and phenotypic data used above and we calculated the association of each single 
Table 2 The 24 female fertility traits analysed

\begin{tabular}{|c|c|c|c|}
\hline Trait group & Trait sub-group & Trait & Description \\
\hline \multirow[t]{5}{*}{ Fertility traits } & \multirow[t]{5}{*}{ Fertility } & Non-return rate heifer (nrr) & Heifers non-return rate after 56 days, binary \\
\hline & & Non-return rate cow (nrk) & Cows non-return rate after 56 days, binary \\
\hline & & Interval first to last insemination heifer (vzr) & Interval between first and last insemination for heifer, days \\
\hline & & Interval first to last insemination cow (vzk) & Interval between first and last insemination for cows, days \\
\hline & & Interval calving to insemination (raz) & Interval from calving to first service, days \\
\hline \multirow[t]{10}{*}{ Birth traits } & \multirow[t]{5}{*}{ Birth history direct } & Percentage normal births (ngd) & Calving ease, scored between 1-without help to 5-dystocia \\
\hline & & Percentage live births (lgd) & Percentage of calves born alive \\
\hline & & Birth weight (ggd) & Weight of calve at birth, kg \\
\hline & & Gestation length (tdd) & Days from successful insemination to birth \\
\hline & & Multiple birth (twind) & Percentage of multiple births \\
\hline & \multirow[t]{5}{*}{ Birth history maternal } & Percentage normal births (ngm) & Calving ease, scored between 1-without help to 5-dystocia \\
\hline & & Percentage live births (lgm) & Percentage of calves born alive \\
\hline & & Birth weight (ggm) & Weight of calve at birth, kg \\
\hline & & Gestation length (tdm) & Days from successful insemination to birth \\
\hline & & Multiple birth (twinm) & Percentage of multiple births \\
\hline \multirow[t]{9}{*}{ Growth-related traits } & \multirow[t]{3}{*}{ Rearing success } & Survival period 1 (p1) & Survival from day 3 up to day 30 \\
\hline & & Survival heifer period 2 (hp2) & Survival of heifers from day 31 up to 458 days \\
\hline & & Survival bull period 2 (bp2) & Survival of young bulls from 31 days up to 183 days \\
\hline & \multirow[t]{3}{*}{ Slaughter traits calves } & Slaughter weight (cwco) & Weight at slaughter, kg \\
\hline & & Carcass conformation score (ccco) & Amount of meat at slaughter, $\mathrm{kg}$ \\
\hline & & Carcass fat score (cfco) & Fat cover in the meat \\
\hline & \multirow[t]{3}{*}{ Slaughter traits adults } & Slaughter weight (cwao) & Weight at slaughter, kg \\
\hline & & Carcass conformation score (ccao) & Amount of meat at slaughter, kg \\
\hline & & Carcass fat score (cfao) & Fat cover in the meat \\
\hline
\end{tabular}

SNP to the deregressed breeding value using the model: $y_{E B V}=\mu+G+S \boldsymbol{\beta}+\epsilon$, where $y_{E B V}$ is the drEBV, $\mu$ is the average of the drEBV, $G$ is a random effect based on a GRM estimated by the method in [45], $S$ is a scalar of the genotype status of each SNP per animal, $\beta$ is the estimated fixed effect of each SNP and $\epsilon$ accounts for random variation. For this analysis, we used the snp1101 software [40] and applied a Bonferroni correction for multiple testing with an increased significance level at $\mathrm{p}<4.35 \mathrm{e}-7$. All the genotyped animals were included in these models. The GWAS results were visualized by creating Manhattan plots with the qqman package [46].

\section{Preparation of the whole-genome sequencing data}

To identify the putative causative variants, we performed whole-genome sequencing on selected animals that were predicted to be carriers of the identified haplotypes. For this purpose, we sequenced 70 animals in addition to the 360 genomes that had been sequenced within other projects or which are publicly available, to reach a total of 430 genomes of healthy breeding animals (see Additional file 1: Table S1). Among these, 114 and 100 were from purebred BS and $\mathrm{OB}$ animals, respectively. First, the reads were trimmed with fastp [47] by applying the following quality thresholds with the flags: --qualified_quality_phred 20,--length_required 35, --cut_window_size 3,--cut_mean_quality 15, --cut_by_quality5 20, --cut_by_quality3 20, and for the WGS data from the NovaSeq6000, the flag --trim_poly_g was added. The quality-controlled reads were mapped to the latest catthe reference sequence ARS-UCD1.2 [36, 37] using the Burrows-Wheeler Aligner v. 0.7.17 [48], deduplicated by using the MarkDuplicates tool from the Picard software v.2.18.2 [49], recalibrated using the BaseRecalibrator and PrintReads applications of the GATK v3.8.1.0.gf15c1c3ef software [50] with known variants from the 1000 Bull Genomes Project run 7 (BQSR file version 2) [51], and sorted by using the samtools v1.8 software [52].

The average read depth and insert size were calculated using covstats in the goleft v0.1.19 tool [53]; read depth ranged from $6.8 \times$ to $72.8 \times$, with an average of 18.6x. For genotyping, the HaplotypeCalling, CombineGVCFs, CatVariants and GenotypeGVCFs tools from GATK v3.8.1.0.gf15c1c3ef were used to produce a variant call format (VCF) file [50]. To predict the effects of the detected variants, we used the NCBI Annotation Release 106 [54] and the SnpEff v4.3 software [55]. Furthermore, quality scores were estimated for each variant 
by applying the GATK recommendation for hard-filtering of indels and small nucleotide variants (SNV) with the SelectVariants and VariantFiltration tools of GATK v3.8.1.0.gf15c1c3ef [50]. This workflow is consistent with the workflow proposed by the 1000 Bull Genomes Project (run 7) [51, 56].

Analysis of the WGS data for the design of the custom array An algorithm was implemented to efficiently screen the identified haplotype regions with a significant depletion in homozygous animals for candidate variants. Thus, we increased the window size by 2 million bp on each side and we looked for variants that did not occur in the homozygous state in any of the 430 animals. As the read depth varied, we allowed for missing values (a maximum of $50 \%$ of genotypes) but assumed that all variants passed the quality score criteria. In addition, we postulated that at least one animal in each population, BS or OB, had to be a carrier of potential variants, but that not more than $75 \%$ of the animals should be carriers.

Subsequently, we designed a custom Affymetrix array called "SWISScow chip" in cooperation with the Swiss breeding associations. We selected 465,768 variants to design this custom array, of which 236,043 were selected from the haplotype regions mapped in this study and 230,305 were protein-coding variants spread genomewide. The design process allowed us to consider $44 \%$ of the initially selected variants. The final SNP panel encompassed 318,216 variants, including 112,854 "routine" variants that were genotyped before using different Illumina bovine beadchips plus 205,362 project-specific variants, so called "research" markers.

This array was used in the Swiss routine genotyping throughout 2020, and provided us with the genomic information of 13,667 animals including 6575 BS and 1489 OB plus a cohort of 5603 Swiss Holstein (HO) cattle.

\section{Linkage disequilibrium (LD) analyses}

To confirm the association between the variants in the VCF file and the defined haplotypes, we calculated the linkage disequilibrium between the defined haplotypes' diplotypes and the variants in the VCF file, and with the genotypes from the custom array. While linkage analysis of the VCF file included 79 BS and 94 OB samples, further analysis depended on the quality control performed on the VCF data of the chromosome of interest (plink function --mind 0.1) [57]. This information was integrated into the data from the custom array, including the 8064 Braunvieh genotypes. Linkage analyses were performed using the plink v1.9 software [57].

\section{Visualization of the results}

To provide a comprehensive overview of the results, the OmicCircos Rpackage was used to visualize the identified genomic regions [58]. Figures 2 and 3 were obtained by including the genomic regions of all significant haplotypes from the trio approach and, if in the same haplotype region, a significant haplotype with the pgp approach was found, this is indicated in the plots. Furthermore, the plots include the LD results between markers on the custom array with the diplotypes, the significant haplotype association for the various traits, and the significant GWAS association combined in the above-defined trait groups (Table 2) for BS and OB, respectively.

\section{Interpretation of the variants}

For the interpretation of the genomic variants, the UCSC Genome Browser tool suite was used to calculate conservation scores [59]. First, the LiftOver UCSC tool and their LiftOver file for BosTau9 to HG38, which is the DNA base conversion from the bovine reference sequence ARS-UCD1.2 [36] to the human genome 38 [60], was applied by command-line coordinate, lifting all the variants from the VCF file. Furthermore, for these new positions, the base-wise conservation scores of 99 vertebrate genomes with the human genome were extracted from the UCSC database and resulted in the phyloP and PhastCons scores shown in Table 3 and Table S5 (see Additional file 6: Table S5) [61, 62]. Both scores represent a comparative genomic alignment approach, but they use different algorithms. PhyloP measures the conservation and evolutionary acceleration at individual sites with absolute values of $-\log$ (p-value), and PhastCons identifies stretches of conservation with values demonstrating probabilities of negative selection from 0 to 1 [61, 62].

For the variants that show a linkage between the WGS data and the haplotypes in relevant regions, we extracted information about their effects by manually using the web-based prediction tools PROVEAN [63] and MutPred2 [64] resulting in the SiftScores and MutPred2 scores shown in Table S5 (see Additional file 6: Table S5).

Finally, we analysed the segregation of candidate variants within the international cattle population of the 1000 Bull Genomes project run 8 that includes 4109 animals from various breeds [56].

\section{Results \\ Detection of numerous novel haplotypes associated with fertility, birth, and growth-related traits}

We identified many genomic candidate regions that show a significant depletion in homozygosity, significant haplotype association, and a genome-wide association to fertility, birth, and growth-related traits. For the 


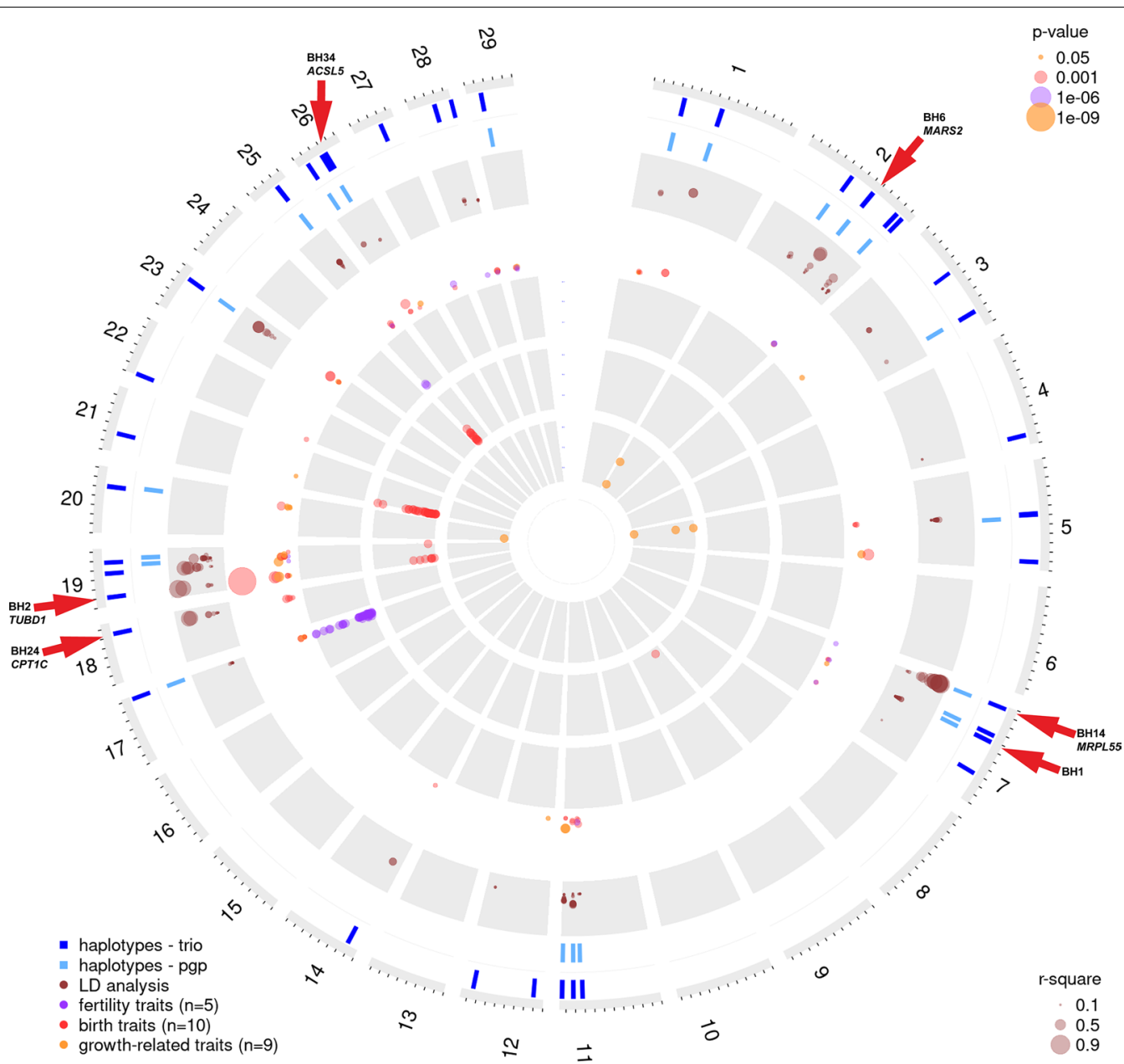

Fig. 2 Genome-wide summary of the data mining for the Brown Swiss population. In the outer circles, the identified haplotypes per chromosome with reduced homozygosity for the trio-based approach are indicated in dark blue and their associated haplotypes of the pgp-based approach in light blue. Note that only the haplotypes that were detected through the pgp-based approach are shown if, within the same region, another haplotype was detected by the trio-based approach. The circle with the brown dots indicates $L D\left(r^{2}\right)$ between haplotypes and markers on the custom SNP array. Note that the dot size correlates with the extent of LD. The third circle shows the significant haplotype association results. Note that the different colors represent the three groups of evaluated traits and the dot size correlates with the significance values accordingly. The three inner circles present the significant GWAS results across all fertility (purple), birth (red), and growth-related (yellow) traits. Scales are based on the $-\log 10$ (p-value). Note that the red arrows indicate the previously identified haplotypes BH1 and BH2 [19] and the herein described MRPL55-related haplotype $\mathrm{BH} 14$, as well as the $\mathrm{BH} 14, \mathrm{BH} 24$ and $\mathrm{BH} 34$ haplotypes and their associated genes that harbor the most likely causative variants

two Braunvieh populations studied here, BS and OB, each of the haplotype analyses based on the pgp- and the trio-based approaches resulted in a concatenated list of all the haplotypes that deviated significantly from HWE. The identified haplotypes were named according to previous studies [19] as BH haplotypes for BS and $\mathrm{OH}$ haplotypes for $\mathrm{OB}$ (Tables 3 and 4). For the BS population, we detected 38 haplotypes with the triobased approach (Table 3) and 53 haplotypes with the pgp-based approach, with 19 overlapping haplotypes (Fig. 2) and (see Additional file 2: Table S2). Two of the identified haplotypes, $\mathrm{BH} 1$ on chromosome 7 and $\mathrm{BH} 2$ on chromosome 19 had already been detected in 2011 [19] (Fig. 2). The haplotypes that deviated most significantly from HWE were $\mathrm{BH} 6, \mathrm{BH} 14$, and $\mathrm{BH} 1$, with no homozygous carriers although at least 44 were expected (see Additional file 2: Table S2). For the OB population, we detected five significant novel haplotypes with the trio-based approach (OH2 to OH6, see Table 4) and three haplotypes with the pgp-based approach, which all overlapped with those detected by the trio-based approach (Fig. 3) and (see Additional file 2: Table S2). The haplotypes that deviated most significantly from HWE were $\mathrm{OH} 2$ and $\mathrm{OH} 4$, with no observed homozygous diplotype 


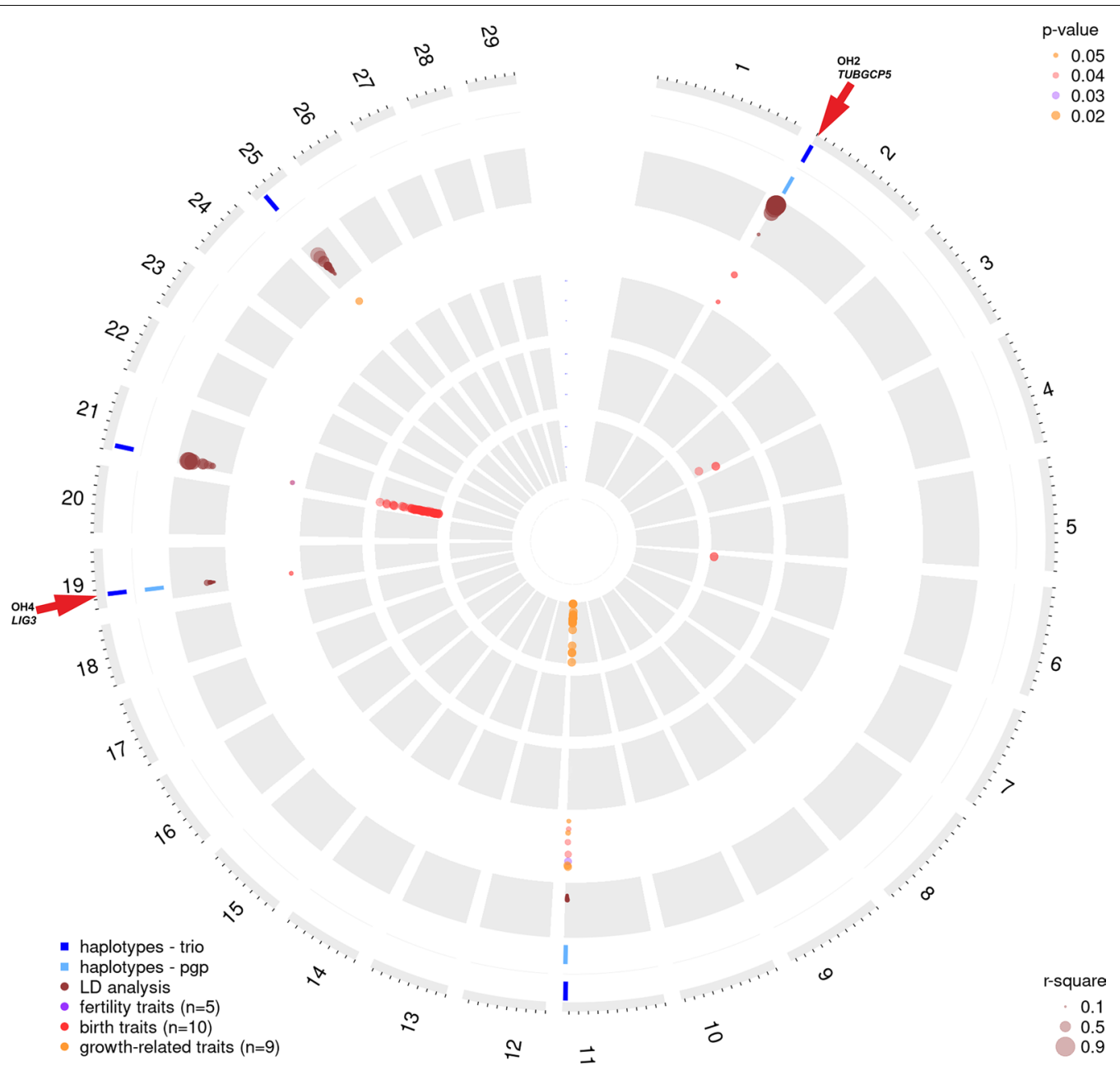

Fig. 3 Genome-wide summary of the data mining for the Original Braunvieh population. In the outer circles, the identified haplotypes per chromosome with reduced homozygosity for the trio-based approach are indicated in dark blue and their associated haplotypes of the pgp-based approach in light blue. Note that only the haplotypes that were detected through the pgp-based approach are shown if, within the same region, another haplotype was detected by the trio-based approach. The circle with the brown dots indicates LD $\left(r^{2}\right)$ between haplotypes and markers on the custom SNP array. Note that the dot size correlates with the extent of LD. The third circle shows the significant haplotype association results. Note that the different colors represent the three groups of evaluated traits and the dot size correlates with the significance values accordingly. The three inner circles present the significant GWAS results across all fertility (purple), birth (red), and growth-related (yellow) traits. Scales are based on the $-\log 10$ (p-value). Note that the red arrows indicate the LIG3-related haplotype $\mathrm{OH} 4$ described in this paper, as well as the $\mathrm{OH} 2$ haplotype and its associated gene TUBGCP5

carriers, although at least 15 were expected (see Additional file 2: Table S2). After correction for FDR, three interesting haplotype regions $(\mathrm{OH} 7, \mathrm{OH} 8$ and $\mathrm{OH} 9)$ with a non-significant depletion of homozygosity were found for OB (see Additional file 2: Table S2; Additional file 3: Table S3; and Additional file 5: Table S4).

The complete GWAS results for the 24 traits, conducted on the identified $\mathrm{BH}$ and $\mathrm{OH}$ haplotypes are in Table S3 (see Additional file 3: Table S3). $\mathrm{BH}$ and $\mathrm{OH}$ haplotypes showed significant associations on 21 and five different chromosomes, respectively (Figs. 2 and 3). The strongest significant association was found for $\mathrm{BH} 2$ with four birth and two growth-related traits (see Additional file 3: Table S3), which confirms the previous finding that the BH2-related missense variant p.His210Arg in the TUBD1 gene causes ill-thrift and juvenile mortality in Brown Swiss cattle [11].

All the results of the single SNP GWAS are visualized in traditional Manhattan plots (see Additional file 4: Fig. S1) and the most significant markers were selected for a comprehensive overview per trait group (Figs. 2 and 3) and (see Additional file 5: Table S4). For example, obvious GWAS hits for fertility traits in BS, such as non-return rate and interval first to last insemination 
Table 3 List of haplotypes in the Brown Swiss population identified by the trio-based approach

\begin{tabular}{|c|c|c|c|c|c|}
\hline Name & $\mathrm{Chr}$ & Start & End & $\begin{array}{l}\text { Allele } \\
\text { frequency }\end{array}$ & $\begin{array}{l}\text { Proposed } \\
\text { associated } \\
\text { gene }^{\mathrm{a}}\end{array}$ \\
\hline $\mathrm{BH} 1^{\mathrm{b}}$ & 7 & 41371808 & 42545291 & 3.02 & TCF3 \\
\hline $\mathrm{BH} 2^{\mathrm{c}}$ & 19 & 9726237 & 10819756 & 4.22 & TUBD1 \\
\hline $\mathrm{BH} 3$ & 1 & 34908448 & 36034631 & 2.05 & \\
\hline $\mathrm{BH} 4$ & 1 & 76587512 & 77861430 & 2.20 & GMNC \\
\hline $\mathrm{BH} 5$ & 2 & 57787148 & 58768161 & 2.87 & $\angle R P 1 B$ \\
\hline $\mathrm{BH} 6$ & 2 & 86065338 & 87460373 & 3.41 & MARS2 \\
\hline $\mathrm{BH} 7$ & 2 & 120284002 & 121473182 & 1.93 & SPATA3 \\
\hline $\mathrm{BH} 8$ & 2 & 127933873 & 129004898 & 2.98 & \\
\hline $\mathrm{BH} 9$ & 3 & 52432936 & 52,689742 & 1.43 & HFM1 \\
\hline $\mathrm{BH} 10$ & 3 & 101636810 & 102827915 & 2.26 & \\
\hline $\mathrm{BH} 11$ & 4 & 108811998 & 109953666 & 2.66 & \\
\hline $\mathrm{BH} 12$ & 5 & 57653479 & 59777422 & 2.32 & $R N F 41$ and $\angle R P 1$ \\
\hline $\mathrm{BH} 13$ & 5 & 110617654 & 111664596 & 1.63 & \\
\hline $\mathrm{BH} 14$ & 7 & 2588873 & 3357718 & 3.04 & MRPL55 \\
\hline $\mathrm{BH} 15$ & 7 & 33954389 & 34956540 & 4.12 & \\
\hline $\mathrm{BH} 16$ & 7 & 79845977 & 80708046 & 2.20 & \\
\hline $\mathrm{BH} 17$ & 11 & 82034654 & 82881173 & 2.39 & PGGHG \\
\hline $\mathrm{BH} 18$ & 11 & 91766016 & 92804942 & 2.54 & \\
\hline $\mathrm{BH} 19$ & 11 & 103891372 & 105003404 & 1.77 & \\
\hline $\mathrm{BH} 2 \mathrm{O}$ & 12 & 10254014 & 10967287 & 2.02 & \\
\hline $\mathrm{BH} 21$ & 12 & 74954939 & 75817277 & 1.61 & \\
\hline $\mathrm{BH} 22$ & 14 & 12290759 & 13350202 & 2.64 & \\
\hline $\mathrm{BH} 23$ & 17 & 69786512 & 70645226 & 2.98 & \\
\hline $\mathrm{BH} 24$ & 18 & 52977041 & 54113281 & 2.32 & CPT1C \\
\hline $\mathrm{BH} 25$ & 19 & 35808547 & 36844178 & 1.86 & SNF8 \\
\hline $\mathrm{BH} 26$ & 19 & 47874708 & 48724082 & 1.89 & \\
\hline $\mathrm{BH} 27$ & 20 & 50058036 & 51370414 & 2.56 & \\
\hline $\mathrm{BH} 28$ & 21 & 19368258 & 20362870 & 1.60 & \\
\hline $\mathrm{BH} 29$ & 22 & 311724 & 1277064 & 2.07 & \\
\hline $\mathrm{BH} 30$ & 23 & 39600366 & 40609442 & 2.49 & \\
\hline BH31 & 25 & 27713801 & 29132187 & 1.80 & ITGAD and SPN \\
\hline BH32 & 26 & 9215188 & 10180467 & 1.72 & \\
\hline $\mathrm{BH} 33$ & 26 & 26659976 & 27689471 & 2.72 & \\
\hline BH34 & 26 & 31353340 & 32429589 & 2.37 & ACSLS \\
\hline BH35 & 27 & 28524504 & 29550764 & 1.80 & \\
\hline $\mathrm{BH} 36$ & 28 & 26661955 & 27743768 & 1.75 & \\
\hline BH37 & 28 & 45215132 & 45913154 & 2.07 & \\
\hline $\mathrm{BH} 38$ & 29 & 14497752 & 15554643 & 3.08 & \\
\hline
\end{tabular}

BH BS haplotype, Chr chromosome number, Start and End start and end positions according to the reference sequence ARS-UCD1.2 [36]

${ }^{\text {a }}$ According to NCBI Annotation Release 106 [54]

${ }^{b}$ Previously described haplotype [19]

${ }^{\text {e }}$ Previously described haplotype and associated gene $[11,19]$

are on chromosome 17 where $\mathrm{BH} 23$ was detected, and very strong GWAS signals for birth traits in $O B$, such as calving ease, birth weight, and gestation length, are
Table 4 List of haplotypes in the Original Braunvieh population identified by the trio-based approach

\begin{tabular}{lrllll}
\hline Name $^{\mathbf{a}}$ & Chr & Start & End & $\begin{array}{l}\text { Allele } \\
\text { frequency }\end{array}$ & $\begin{array}{l}\text { Proposed } \\
\text { associated } \\
\text { gene }\end{array}$ \\
\hline $\mathrm{bH} 2$ & 2 & 1005580 & 1614673 & 4.48 & TUBGCP5 \\
$\mathrm{OH} 3$ & 11 & 10,406494 & 104418358 & 5.76 & MYMK \\
$\mathrm{OH} 4$ & 19 & 14336760 & 15222429 & 3.87 & LIG3 \\
$\mathrm{OH} 5$ & 21 & 5195518 & 6367707 & 4.56 & LYSMD4 \\
$\mathrm{OH} 6$ & 25 & 9596610 & 10624288 & 3.87 & USP7 \\
\hline
\end{tabular}

$\mathrm{OH}$ OB haplotype, $\mathrm{Chr}$ chromosome number, Start and End start and end positions according to the reference sequence ARS-UCD1.2 [36]

a Since the name $\mathrm{OH} 1$ is reserved for a pathogenic CNGB3-variant [101], numbering starts at $\mathrm{OH} 2$

${ }^{\mathrm{b}}$ According to NCBI Annotation Release 106 [54]

on chromosome 21, where OH5 was found. Among the studied growth-related traits, we identified a highly significant association signal on chromosome 11 in $\mathrm{OB}$, which affects carcass conformation and co-localizes with the OH3 haplotype.

\section{Potential candidate causative variants in MRPL55 and LIG3}

Subsequently, we mined the genome-wide sequence data to identify potential causative candidate variants responsible for either prenatal lethality or postnatal lethality, or sub-lethal phenotypes. Analysis of the variants that show depletion in homozygosity and are in LD with the haplotype identified protein-changing variants that are potentially causative in five $\mathrm{BH}$ and two $\mathrm{OH}$ haplotypes (Tables 5 and 6). Genotyping based on the SWISScow custom array allowed to validate whether three of the BS variants were present in several thousands of young animals. As for the haplotypes, we detected animals with homozygous variant genotypes in the current population, which lead us to anticipate either that late-onset undesired traits might occur or that these variants have incomplete penetrant effects. The disorders known to be associated with the affected genes range from embryonic lethality (e.g., MRPL55 [65] and LIG3 [66]) and metabolic diseases (e.g. acyl-CoA synthetase long chain family member 5 (ACSL5) [67]) to neurodevelopmental disorders (e.g., methionyl-tRNA synthetase 2 (MARS2) [68]) (Tables 5 and 6).

We specifically selected one variant on chromosome 7 at $\sim 3 \mathrm{Mb}$ in the BS population that is predicted to be deleterious due to a stop-gain variant in the MRPL55 gene. This mutation introduces a premature stop codon (p.Arg57*), which truncates the encoded protein by almost $80 \%$ (Table 5). This variant is in perfect $L D\left(r^{2}=1\right)$ with the BH14 haplotype in the WGS animals and in high $\mathrm{LD}\left(\mathrm{r}^{2}=0.9\right)$ in the custom array genotyped animals. It 
Table 5 Short list of five potential candidate causative variants for the Brown Swiss population ${ }^{\text {a }}$

\begin{tabular}{|c|c|c|c|c|c|c|c|c|}
\hline \multicolumn{2}{|c|}{$\begin{array}{l}\text { Haplotype-region } \\
\text { information }\end{array}$} & \multirow[t]{2}{*}{ Gene } & \multirow[t]{2}{*}{ OMIM/OMIA } & \multirow{2}{*}{$\begin{array}{l}\text { Associated } \\
\text { disorder/gene } \\
\text { function }\end{array}$} & \multicolumn{4}{|c|}{ Variant designation $^{\mathbf{b}}$} \\
\hline Name & Approach & & & & $\begin{array}{l}\text { Genomic } \\
\text { position }\end{array}$ & Transcript & $\begin{array}{l}\text { Coding DNA } \\
\text { change }\end{array}$ & Protein change \\
\hline $\mathrm{BH} 2$ & Trio & $T U B D 1^{C}$ & $\begin{array}{l}\text { 607344/001939- } \\
9913\end{array}$ & Juvenile mortality & chr19:10833921 & NM_001075470.2 & c. $629 A>G$ & p.His210Arg \\
\hline $\mathrm{BH} 6$ & Trio and pgp & MARS2 & 609728 & $\begin{array}{l}\text { Spastic ataxia } 3 \\
\text { (lethal) }\end{array}$ & chr2:86191230 & NM_001098971.1 & c. $1553 \mathrm{G}>\mathrm{A}$ & p.Arg518Gln \\
\hline $\mathrm{BH} 14$ & Trio and pgp & MRPL55 & 611859 & $\begin{array}{l}\text { Early pregnancy } \\
\text { loss }\end{array}$ & chr7:2996436 & NM_001303490.1 & c. $169 C>T$ & p.Arg $57^{*}$ \\
\hline $\mathrm{BH} 24$ & Trio & CPT1C & 608846 & $\begin{array}{l}\text { Spastic paraplegia } \\
\text { (lethal) }\end{array}$ & chr18:56098048 & XM_002695120.5 & c.158G $>A$ & p.Gly53Asp \\
\hline $\mathrm{BH} 34$ & Trio & ACSL5 & $\begin{array}{l}605677 / 002226- \\
9615\end{array}$ & $\begin{array}{l}\text { Lipid malabsorp- } \\
\text { tion }\end{array}$ & chr26:32940521 & NM_001075650.1 & c. $528 C>G$ & p.Asn176Lys \\
\hline
\end{tabular}

Chr chromosome, $\mathrm{BH}$ BS haplotype

${ }^{\text {a }}$ Comprehensive list available in Table S5 (see Additional file 6: Table S5)

${ }^{\mathrm{b}}$ According to the reference sequence ARS-UCD1.2 [36] and NCBI Annotation Release 106 [54]

cPreviously described variant [11]

Table 6 Short list of two potential candidate causative variants for the Original Braunvieh population ${ }^{\text {a }}$

\begin{tabular}{|c|c|c|c|c|c|c|c|c|}
\hline \multicolumn{2}{|c|}{$\begin{array}{l}\text { Haplotype-region } \\
\text { information }\end{array}$} & \multirow[t]{2}{*}{ Gene } & \multirow[t]{2}{*}{ OMIM/OMIA } & \multirow{2}{*}{$\begin{array}{l}\text { Associated } \\
\text { disorder/gene } \\
\text { function }\end{array}$} & \multicolumn{4}{|c|}{ Variant designation $^{b}$} \\
\hline Name & Approach & & & & $\begin{array}{l}\text { Genomic } \\
\text { position }\end{array}$ & Transcript & Coding DNA change & Protein change \\
\hline $\mathrm{OH} 2$ & Trio and pgp & TUBGCP5 & 608147 & $\begin{array}{l}\text { Proper formation } \\
\text { of the mitotic } \\
\text { spindles }\end{array}$ & chr2:1268426 & NM_001102495.1 & C. $311 C>A$ & p.Thr104Lys \\
\hline $\mathrm{OH} 4$ & Trio and pgp & LIG3 & 600940 & $\begin{array}{l}\text { Embryonic } \\
\text { lethality }\end{array}$ & chr19:15080335 & NM_001038107.2 & $\begin{array}{l}\text { c. } 2483 \_2484+4 \text { delAGG } \\
\text { TGC }\end{array}$ & p.Lys $828 f \mathrm{fs}$ \\
\hline
\end{tabular}

Chr chromosome, $\mathrm{OH} O \mathrm{OB}$ haplotype

${ }^{a}$ Comprehensive list available in Table S5 (see Additional file 6: Table S5)

${ }^{\mathrm{b}}$ According to the reference sequence ARS-UCD1.2 [36] and NCBI Annotation Release 106 [54]

was never observed in the homozygous state in any of the BS individuals. Furthermore, BH14 shows a significant negative association with the fertility trait interval first to last insemination' in cows and a mentionable but non-significant negative association with the fertility trait 'interval calving to first insemination' and the growthrelated trait 'survival of young bulls' (see Additional file 3: Table S3). Evaluation of the MRPL55-related variant with the custom array revealed that it segregates within the BS population at a frequency of 0.032 without any homozygous carriers and deviates significantly from HWE (see Additional file 6: Table S5). In the OB population with more than 1400 animals genotyped with the SWISScow custom array, genotyping found only two heterozygous animals for this variant. In addition, none of the 4109 animals from various breeds within the run 8 data of the 1000 Bull Genomes project were homozygous for this variant [51].
In the BS population, we identified three additional missense variants in the carnitine palmitoyltransferase 1C (CPT1C), MARS2, and ACSL5 genes (Table 5), which affect highly conserved nucleotides. Their effects on protein level were predicted to be deleterious by PROVEAN and pathogenic by MutPred2 (see Additional file 6: Table S5). Unfortunately, only one of these variants was included in the SWISScow custom array, which was associated with $\mathrm{BH} 24$ and located in the CPT1C gene (p.Gly53Asp). This variant was never detected in the homozygous state in the WGS data, and only a few homozygous carrier animals were detected in the SWISScow genotypes (see Additional file 6: Table S5). The $\mathrm{BH} 24$ haplotype is associated with several birth traits such as birth weight, percentage of normal births, and percentage of live births. The p.Arg518Gln variant in the MARS2 gene that is associated with the BH6 haplotype is also of interest since this haplotype was never detected in 
the homozygous state. Unfortunately, this variant was not considered in subsequent array-based genotyping. Lastly, the p.Asn176Lys variant in the ACSL5 gene that is associated with the $\mathrm{BH} 34$ haplotype was also never detected in the homozygous state within the available WGS data and, to date, has not been genotyped in other animals. BH34 was rarely observed in the homozygous state in the BS population and is statistically negatively associated with the growth-related traits 'heifer survival after 30 days' and 'carcass fat score in calves'.

In the $\mathrm{OB}$ population, we draw attention to the haplotype region OH4 located on chromosome 19 at $\sim 15 \mathrm{Mb}$. For this haplotype, we identified a frameshift variant (p.Lys828fs) in the LIG3 gene leading to the change of 90 residues and premature termination of translation compared to the wild-type protein sequence, which is 26 residues longer (Table 6). This variant is in perfect $L D$ $\left(\mathrm{r}^{2}=1\right)$ with the OH4 haplotype in the WGS animals. $\mathrm{OH} 4$ is negatively associated with the percentage of live births. Unfortunately, this frameshift variant was not part of the SWISScow custom array. Nevertheless, the LIG3 variant segregates at an allele frequency of 0.035 in the WGS data of Braunvieh animals. In addition, none of the 4109 animals from various breeds within the run 8 data of the 1000 Bull Genomes project [51] were homozygous for this variant. According to OMIM \#600940, the LIG3 gene is essential for DNA repair of mitochondrial DNA.

A missense variant that affects an evolutionary highly conserved residue (p.Thr104Lys) in the tubulin gamma complex associated protein 5 (TUBGCP5) gene was detected in the OB population but was never observed in the homozygous state in the WGS data (see Additional file 6: Table S5). The associated $\mathrm{OH} 2$ haplotype has a negative effect on multiple births and a positive effect on the percentage of normal births. Although absent from the SWISScow array, this variant segregates at an allele frequency of 0.021 in the WGS data and is in perfect LD with the haplotype (see Additional file 6: Table S5).

In addition, for 13 other $\mathrm{BH}$ and two other $\mathrm{OH}$ haplotypes, we propose candidate causative variants (see Additional file 6: Table S5), but most of them are predicted to have a neutral or benign effect or represent synonymous variants.

\section{Discussion}

This comprehensive study explored the genomic data of the two current Swiss Braunvieh dairy populations BS and $\mathrm{OB}$, for reduced homozygosity due to hidden recessive variants. Such variants which, for the most part, change the amino acid sequence of proteins, lead to natural or artificial selection against homozygous individuals. This phenomenon could be due to embryonic lethality, reduced rearing success, or exclusion from the breeding population due to poor development. To better understand the functional role of the associated haplotype regions, we performed genome-wide missing homozygosity scans and subsequent comprehensive association analyses.

In the recent past, various studies that aimed at pinpointing such haplotype regions used routine SNP genotyping data and the standard approach (pgp) in which the paternal grandfather replaces the usually non-genotyped dam $[19,21,27]$. Here, we chose a trio-based approach, assuming that it would increase the power of the statistical analysis of haplotypes due to the direct relationships of the families analyzed [21]. The trio-based approach resembles the transmission ratio distortion approach used to search for lethal alleles, however, the latter is based on Bayesian statistics, while we applied the Fisher exact test of HWE [7, 8, 24, 41]. Especially for the OB population for which the amount of available data is limited, the trio-based approach is notably more efficient than the standard analysis, since more regions with lower haplotype frequencies were detected. In contrast, for the BS population, the pgp-based approach, which considers more than twice the number of family groups, detected more regions than the trio-based analysis. However, it should be kept in mind that an increased number of detected haplotype regions might include more false positives, which is supported by our results with some of the haplotype regions detected in the BS population not showing any phenotypic associations. Therefore, we focused on the haplotype regions that were detected by the trio-based approach and showed phenotypic associations with the 24 analyzed fertility, birth, and growthrelated traits.

To validate the chosen approach, we searched for previously identified haplotypes and known causal recessive variants and confirmed the $\mathrm{BH} 1$ and $\mathrm{BH} 2$ haplotypes in the studied BS population [11, 19]. BH2 is known to be negatively associated with fertility and growth-related traits. We also confirmed that the variant in the TUBD1 gene showed the highest level of LD with $\mathrm{BH} 2$. Interestingly, although breeding against this variant has been practiced for several years, we were still able to detect this haplotype region and its effects on the studied traits. However, strong selection of the bulls has successfully eliminated the two inherited disorders spinal muscular atrophy $[69,70]$ and weaver syndrome $[14,31,71]$ since we did not detect any carriers. Regarding the OB population, it is known that the Fleckvieh haplotype 2 (FH2) and the associated frameshift variant in the $S L C 2 A 2$ gene segregate in the OB population at an allele frequency of 0.05 and cause the Fanconi-Bickel syndrome with growth retardation $[9,72]$. Nonetheless, we did not detect the region surrounding this variant on chromosome 1 . The 
reason why we could not find a significant depletion in homozygosity for $\mathrm{FH} 2$ was that the genotyping data included several homozygous carriers of the variant associated with the Fanconi-Bickel syndrome. Due to the fact that this disorder is non-lethal, but induces liver and kidney defects leading to reduced growth [9], apparently normally developed new-born calves are genotyped before the manifestation of growth retardation.

Our aim was to detect novel causative variants, but first we confirmed the detection of the BH1 haplotype region previously reported in BS cattle $[19,33]$. We found evidence for depletion of homozygotes, but a positive association with the growth-related trait carcass fat score and no indication of a negative effect on fertility traits as previously described [33]. We suggest that it is caused by the p.Asp70Asn missense variant that affects a highly conserved nucleotide in the transcription factor 3 (TCF3) gene, also known as E2A. In men, mutations in this gene are associated with agammaglobulinemia 8 (OMIM \#616941) (see Additional file 6: Table S5). Tcf3 knock-out mice show growth retardation and increased neonatal death [73]. TCF3 is associated with the Wnt signaling pathway and thereby influences osteogenesis and is essential for B cell differentiation [73, 74]. This same variant was previously reported to be associated with BH1, but with inconclusive results [33]. In our study, the estimated LD between $\mathrm{BH} 1$ and this variant is rather low and the haplotype associations are merely suggestive. Therefore, either this variant causes retarded growth and increased pre-weaning lethality, but with incomplete penetrance, or is irrelevant as not all lossof-function variants have a pathogenic effect [75]. We found several dozens of homozygous carriers of the TCF3 variant, although it segregates with an allele frequency of 0.11 and deviates significantly from HWE. Therefore, we recommend to keep track of this variant, e.g. by clinically examining live homozygous individuals.

Regarding our extensive list of newly identified haplotype regions in the Swiss Braunvieh populations studied here, we propose six candidate causative variants (Tables 5 and 6), which are all supported by high LD values with the corresponding haplotype, and 17 variants of interest (see Additional file 6: Table S5). In the future, for many other mapped haplotypes, the detection of such variants should be done with the same approach based on population-wide evaluation, because unfortunately only every second variant is technically designable for the custom array. Furthermore, it is disputable if instead of array-based genotyping with its known limitations, one could consider genotyping by sequencing by using low-pass sequencing [76, 77]. Nonetheless, this recent method has also strong limitations such as a restricted probability to see the variant of interest on the sequence of a given individual as well as the need to be followed by imputation that is not accurate for recent variants due to the existence of ancestral and derived versions of identical haplotypes. Due to the large evidence gained by the analysis of massive phenotypic and genomic data, we recommend further evaluation of such haplotype regions and candidate variants in the future.

Among the variants suggested to cause reduced omozygosity in the BS population, we highlighted the MRPL55 nonsense variant on bovine chromosome 7 associated with BH14. Statistically, this variant fits perfectly with the expectations for embryonic lethal variants, since LD is very high and there are no homozygous animals. The MRPL55 gene belongs to the mitochondrial ribosomal protein (MRP) gene family, more precisely to the MRPL group, which are genes that make up the large subunit of the mitoribosome complex [78]. The mitoribosome complex is a multi-gene complex of diverse genes, denoted MRP genes [65]. Mouse knock-out experiments have revealed that different genes of the MRP family cause early embryonic death at the stage of pre-gastrulation, and expression analyses of MRP genes have shown their essential role during embryogenesis [65, 79]. Furthermore, mRpL55 was found to have a crucial role during the development of fruit flies, with mRpL55 null individuals showing increased mortality, reduced growth and activity after hatching [80]. Due to the nature of the bovine variant, two-thirds of the truncated protein are lacking, with most likely non-sense mediated decay taking place, thus it can be assumed that the variant leads to a loss-of-function of MRPL55 in the homozygous state. Finally, this could explain putative early embryonic lethality and is consistent with the complete lack of homozygous animals. Moreover, the described biological effect is supported by the association study indicating negative associations with fertility traits and a reduction in heifer survival. The latter can be explained with fertility issues being one of the major culling reasons in dairy cattle [81, 82].

For the BS population, we propose three additional variants as candidate causal variants impairing postnatal survival. First, the BH24-associated missense variant in the CPT1C gene, which is known to be important for the proper development of the brain [83], was predicted to have a deleterious effect on protein level and effects on an evolutionary highly conserved nucleotide. Disorders associated with pathogenic $C P T 1 C$ variants cause lethal spastic paraplegia (OMIM \#616282). The BH24 haplotype shows significant associations with several birth traits and therefore this variant represents a plausible candidate for reduced homozygosity, which was confirmed after targeted genotyping. Second, the BH6associated most likely pathogenic missense variant in 
$M A R S 2$, a gene that plays an important role in embryonic development, is analogous to MRPL55, potentially leading to developmental arrest during early embryogenesis [79]. In humans, variants in this gene are associated with mitochondrial respiratory chain disorders and lead to retarded growth and hypotonia [84], and are also associated with a neurodevelopmental disorder (OMIM \#611390) [68]. Third, the most likely pathogenic BH34associated missense variant in ACSL5, which is involved in lipid metabolism, might cause a developmental delay due to disturbed fat metabolism in various mammalian species (OMIM \#605677, OMIA \#002226-9615) [67], and might explain the negative effects that we observed on growth-related traits. Both the MARS2 and ACSL5 variants need to be evaluated by further genotyping to confirm the postulated effects.

For the $\mathrm{OB}$ population, we propose two novel variants as candidate causal variants impairing embryonic survival. First, the $\mathrm{OH} 4$-associated frameshift variant in the LIG3 gene, which is related to early embryonic lethality in mice [66], is of high interest. Functionally, LIG3 is a DNA binding ligase that is responsible for the repair of strand breaks $[85,86]$. However, more precisely, the function of LIG3 is not essential for the repair of nuclear DNA since other proteins can compensate for the lack of LIG3, but it is essential for the repair of mitochondrial DNA [85, 86]. Second, the $\mathrm{OH} 2$-associated missense variant in the TUBGCP5 gene, although not predicted as having a damaging effect on an evolutionary highly conserved nucleotide, it might represent a suitable candidate, since TUBGCP5 plays an important role in the $\gamma$-tubulin ring complex which binds to the centrosome and thereby affects the cell cycle [87-89]. This complex has a highly conserved structure including six $\gamma$-tubulin proteins. TUBGCP5, in contrast to the other proteins, is present in single copy in the $\gamma$-tubulin ring complex [87-89]. In humans, an association between a TUBGCP5 missense variant and microcephaly, which is a severe anomaly, has been shown [90]. Although to date these two variants have not been evaluated in the broader population, the observed significant negative effect of the $\mathrm{OH} 2$ haplotype on the maternal birth trait 'percentage of live births' and the deleterious nature of the LIG3 and TUBGCP5 variants support the assumed negative impact on reproduction.

Furthermore, three additional haplotypes $(\mathrm{OH} 7, \mathrm{OH} 8$, and $\mathrm{OH} 9$ ) which showed no significant HWE-deviation after correcting for FDR were detected. Interestingly, we found very convincing candidate variants for these haplotypes, such as the missense variant in the HSD3B7 gene located on bovine chromosome 25 . This variant is in high LD with $\mathrm{OH} 9$ and no homozygous carrier animals were found. $H S D 3 B 7$ is associated with the often lethal recessively inherited bile acid synthesis defect (BASD; OMIM \#607764) and with progressive liver disease characterized by malabsorption of lipids and vitamins [91, 92]. HSD3B7 knock-out mice showed increased mortality of homozygous newborns and decreased cholesterol absorption in the surviving animals [92]. Given the nature of the bovine $H S D 3 B 7$ missense variant and the observed negative effect on the growth-related trait 'bull survival,' we speculate that homozygous mutant animals are most likely either non-viable or impaired in development.

Interestingly a unique haplotype region on bovine chromosome 11 was mapped in both populations, suggesting a common causative variant and therefore underlining the common heritage of the two Swiss Braunvieh cattle populations [34, 35]. Nevertheless, previous studies in OB have identified this region as a signature of selection $[35,93]$. For these haplotypes (BH19 and OH3), we have not identified any potentially causative variant. The most probable variant identified was a missense variant in the myomaker (MYMK) gene; however, this variant occurs in the homozygous state in many animals. Therefore, it is likely a less suitable candidate for the depletion of homozygosity. Interestingly the OB-specific GWAS results for the growth-related carcass conformation score traits pinpoint the same region on chromosome 11 at $104 \mathrm{Mb}$. Based on the function of the $M Y M K$ gene on skeletal muscle development [94], we suggest that the impact of this variant on economically important growth traits needs to be subsequently evaluated. In contrast, deletion of Mymk in mice is perinatal lethal [94]. This example illustrates nicely the case of a variant that potentially underlies balancing selection as shown previously in pigs and cattle $[95,96]$. Alternatively, and more likely, other undetected variants, such as more complex structural variants, might be responsible for the observed depletion in homozygosity in this genomic region.

As described above, not all the variants that we claim to be potentially causing disease could be evaluated in the current population, as many of them were unfortunately not included on the custom array. There are technical reasons why many variants were not included on this final array, such as interference with adjacent variants, high GC-content, repetitive DNA, multi-allelic variation, etc. Nonetheless, the SWISScow custom chip proved to be a very powerful and efficient method to validate the segregation of the most likely causative variants in the current population. Furthermore, the genomic data of a population changes over time since the haplotype frequencies can change due to the impact of popular sires. Regarding the haplotype regions, we may have failed to detect some specific haplotype regions because the window size was set at 50 markers, since Hoff et al. 
[25] showed that the homozygosity rate negatively correlates with window length.

Although we propose 24 new variants, including six of high importance, the causal variants for numerous haplotype regions including $\mathrm{BH} 1$ remain unclear. For example, in the BS population, we identified the highly HWE-deviating haplotype $\mathrm{BH} 23$ on chromosome 17 , which showed significant haplotype associations with growth-related and birth traits, plus suggestive associations with fertility. In parallel, for the same region, suggestive GWAS associations with the fertility trait 'non-return rate' were observed, but we failed to identify candidate causal variants.

This analysis is based on the latest reference sequence ARS-UCD1.2 of a Hereford cow, however, the reference sequence still spans many gaps and includes more than 2000 unplaced scaffolds that could potentially harbor important coding sequences [37]. Recently, it was shown that reference graphs based on sequence data of several breeds can include new functional sequences [97]. Another drawback of our approach is that we restricted our analysis to coding variants, while non-coding variants impairing the expression of a protein or more complex structural variants disturbing the function of genes might also be causative. The interference of non-detected structural variants could also be an explanation for the rather low LD values in some regions [98, 99]. Furthermore, as a few homozygous diplotype carriers are alive, some haplotypes may arise due to the high selective pressure on traits within the breeding program. As shown by the example of cholesterol deficiency in Holstein cattle, the associated haplotype can segregate in the population under two indistinguishable versions, a derived variant and its ancestral version [100]. In summary, the reported haplotype regions might reflect population-specific characteristics of the breeding programs, such as the intensive use of individual bulls by artificial insemination or by natural service, a common practice in Swiss OB. Most likely, the focus applied on monogenic Mendelian disorders is too simplistic since a polygenic architecture can often be assumed. The latter is supported by the GWAS results that highlight numerous additional associated genomic regions, which are not affected by a depletion of homozygosity.

\section{Conclusions}

For the first time, we applied the trio-based mapping approach in cattle for the genome-wide detection of haplotypes showing reduced homozygosity that segregate at low to moderate frequencies. We present a short list of potentially causative variants and highlight four coding variants for the Brown Swiss population and two for the Original Braunvieh population located in candidate genes that are involved in embryonic and pre-weaning lethality. Although it will be challenging to evaluate all the candidate variants that we propose here by targeted monitoring of at risk matings and possible clinical examination of live homozygotes, it is important to rule out causality. This study illustrates the difficulty to select for improved fertility and rearing success by focusing on monogenic disorders, since our GWAS results confirmed the polygenic nature of these traits. Nonetheless, the proposed candidate causative variants will help to refine DNA-based selection decisions to improve female fertility and rearing success in Swiss Braunvieh cattle.

\section{Abbreviations}

ACSL5: Acyl-CoA synthetase long chain family member 5; Arg: Arginine; BASD: bile acid synthesis defect; $\mathrm{BH}$ : Brown Swiss Haplotype; $\mathrm{BH}$ : Brown Swiss Haplotype 1; BH2: Brown Swiss Haplotype 2; bp: base pairs; BS: Brown Swiss; CPT1C: Carnitine palmitoyltransferase 1C; Cys: Cysteine; drEBV: deregressed estimated breeding value; FDR: False discover rate; FH2: Fleckvieh Haplotype 2; GS: genomic selection; GWAS: genome-wide association study; HD: high density SNP panel; HO: Holstein; HSD3B7: Hydroxy-delta-5-steroid dehydrogenase, 3 beta- and steroid delta-isomerase 7; HWE: Hardy-Weinberg equilibrium; LD: Iow density SNP panel; LIG3: DNA ligase 3; MARS2: Methionyl-tRNA synthetase 2, mitochondrial; Mb: Mega bases; MRPL55: Mitochondrial ribosomal protein L55; MYMK: Myomaker; OB: dual-purpose breed Original Braunvieh; $\mathrm{OH}$ : Original Braunvieh Haplotype; pgp: parent - grant parent (paternal half-sib groups); QTL: quantitative trait loci; r2: R-squared value of the LD analysis; SCFD2: Sec1 family domain containing 2; SLC2A2: Solute carrier family 2 member 2; SNV: small nucleotide variants; SNP: single nucleotide polymorphism; SNP array: genotyping arrays including single nucleotide polymorphisms; TCF3: Transcription factor 3; TRD: transmission ratio distortion; TUBD1: Tubulin delta 1; TUBGCP5: Tubulin gamma complex associated protein 5; VCF: variant call format file; WGS: whole-genome sequencing.

\section{Supplementary Information}

The online version contains supplementary material available at https://doi. org/10.1186/s12711-021-00686-3.

Additional file 1: Table S1. WGS sample information. All whole-genome sequencing samples included in this study and their project and sample ids from the European Nucleotide Archive (ENA). In addition, the sex, breed and average read depth and insert size are provided.

Additional file 2: Table S2. Haplotype information. All haplotypes for the trio- and pgp-based approaches for the BS (A) and the OB population (B). The descriptive data include the type of analysis, genomic positions, number of expected and observed animals, haplotype frequency, $p$-value from exact tests of Hardy-Weinberg equilibrium and the adjusted p-value according to the Benjamini-Yekutieli procedure.

Additional file 3: Table S3. Output of the haplotype analyses. Results of the estimation of the haplotype effect with different conformation, production and reproduction traits for the BS (A) and OB populations (B).

Additional file 4: Figure S1. Manhattan plots and their QQ-plots of the GWAS results for the BS and OB populations. There is a page for each of the fertility, birth and growth-related trait groups, including a Manhattan plot for every single trait according to Table 2 .

Additional file 5: Table S4. GWAS results. Significant $(p<4.35 e-7)$ GWAS results for the $\mathrm{BS}(\mathbf{A})$ and $(\mathbf{B}) \mathrm{OB}$ populations.

Additional file 6: Table S5. Comprehensive list of candidate causal variants. The data for BS (A) and OB (B) are provided, with information regarding haplotypes and the potential candidate causal variants based on the 
reference sequence ARS-UCD1.2 [36] and the NCBI Annotation Release 106 [54], including variant effect predictions (siftScore [63] and MutPred2 score [64]) and base conservation scores (phyloP and phastCons [61, 62]). Furthermore, the genotype distributions across whole-genome sequencing and genotyping data are included.

\section{Acknowledgements}

The authors are grateful to the Swiss cattle breeding association Braunvieh Schweiz for providing the genomic SNP array and the phenotypic data. We are grateful to Swissgenetics and SelectStar for sending sperm samples of the sires. The assistance of Nathalie Besuchet-Schmutz with DNA extraction is acknowledged. We acknowledge the assistance of the Next Generation Sequencing Platform of the University of Bern for performing the wholegenome re-sequencing experiments. Furthermore, we thank the Interfaculty Bioinformatics Unit of the University of Bern for providing high performance computing infrastructure. The 1000 Bull Genomes Project consortium (Christy Vander Jagt, Hans Daetwyler) is acknowledged for providing the variant catalogue. Christine F. Baes assisted in proofreading.

\section{Authors' contributions}

IMH prepared and analyzed the whole-genome sequencing data, analyzed and interpreted all the obtained results. Furthermore, IMH drafted this manuscript including tables and visualizations. CD acquired the whole-genome sequencing data. MS and FRS handled and analyzed the SNP genotyping data, including the genome-wide association studies and haplotype analysis. CD and FRS designed and supervised the project. All authors read and approved the final manuscript.

\section{Funding}

This study was funded by the Swiss National Science Foundation, grant number 172911. Schweizerischer Nationalfonds zur Förderung der Wissenschaftlichen Forschung, 172911, Cord Drögemüller

\section{Availability of data and materials}

For access to the genotyping data, interested people are asked to contact the Braunvieh breeding association directly.

\section{Declarations}

Ethics approval and consent to participate

According to the Swiss laws, no ethics approval was needed. In this study, already available data was reused and mined by using novel approaches.

\section{Consent for publication}

Not applicable.

\section{Competing interests}

The authors declare that there have no competing interests.

\section{Author details}

'Institute of Genetics, Vetsuisse Faculty, University of Bern, Bremgartenstrasse 109a, 3001 Bern, Switzerland. ${ }^{2}$ Qualitas AG, 6300 Zug, Switzerland.

Received: 11 May 2021 Accepted: 26 November 2021

Published online: 16 December 2021

\section{References}

1. Miglior F, Fleming A, Malchiodi F, Brito LF, Martin P, Baes CF. A 100-year review: Identification and genetic selection of economically important traits in dairy cattle. J Dairy Sci. 2017;100:10251-71.

2. Pryce JE, Woolaston R, Berry DP, Wall E, Winters M, Butler R, et al. World trends in dairy cow fertility. In Proceedings of the 10th World Congress on Genetics Applied to Livestock Production. 18-22 August 2014; Vancouver. 2014.
3. Walsh SW, Williams EJ, Evans ACO. A review of the causes of poor fertility in high milk producing dairy cows. Anim Reprod Sci. 2011:123:127-38.

4. Pryce JE, Royal MD, Garnsworthy PC, Mao IL. Fertility in the high-producing dairy cow. Livest Prod Sci. 2004;86:125-35.

5. Goddard ME, Hayes BJ, Meuwissen THE. Genomic selection in livestock populations. Genet Res (Camb). 2010;92:413-21.

6. Hayes BJ, Bowman PJ, Chamberlain AJ, Goddard ME. Invited review: genomic selection in dairy cattle: progress and challenges. J Dairy Sci. 2009:92:433-43.

7. Casellas J, Cañas-Alvarez JJ, González-Rodríguez A, Puig-Oliveras A, Fina M, Piedrafita J, et al. Bayesian analysis of parent-specific transmission ratio distortion in seven Spanish beef cattle breeds. Anim Genet. 2017:48:93-6

8. Casellas J, Gularte RJ, Farber CR, Varona L, Mehrabian M, Schadt EE, et al. Genome scans for transmission ratio distortion regions in mice. Genetics. 2012;191:247-59.

9. Pausch H, Schwarzenbacher H, Burgstaller J, Flisikowski K, Wurmser C, Jansen S, et al. Homozygous haplotype deficiency reveals deleterious mutations compromising reproductive and rearing success in cattle. BMC Genomics. 2015;16:312.

10. Cole JB, VanRaden PM, Null DJ, Hutchison JL, Cooper TA, Hubbard SM. Haplotype tests for recessive disorders that affect fertility and other traits. Animal Improvement Program Research Report. 2018. https:// www.aipl.arsusda.gov/reference/recessive_haplotypes_ARR-G3.html. Accessed 2021 Apr 15.

11. Schwarzenbacher $\mathrm{H}$, Burgstaller J, Seefried FR, Wurmser C, Hilbe M, Jung $S$, et al. A missense mutation in TUBD1 is associated with high juvenile mortality in Braunvieh and Fleckvieh cattle. BMC Genomics. 2016;17:400.

12. Sonstegard TS, Cole JB, VanRaden PM, Van Tassell CP, Null DJ, Schroeder SG, et al. Identification of a nonsense mutation in CWC15 associated with decreased reproductive efficiency in Jersey cattle. PLoS One. 2013;8:e54872.

13. Charlier C, Agerholm JS, Coppieters W, Karlskov-Mortensen P, Li W, de Jong $\mathrm{G}$, et al. A deletion in the bovine FANCI gene compromiss fertility by causing fetal death and brachyspina. PLoS One. 2012;7:e43085.

14. McClure M, Kim E, Bickhart D, Null D, Cooper T, Cole J, et al. Fine mapping for weaver syndrome in Brown Swiss cattle and the identification of 41 concordant mutations across NRCAM, PNPLA8 and CTTNBP2. PLoS One. 2013;8:e59251.

15. Schütz E, Wehrhahn C, Wanjek M, Bortfeld R, Wemheuer WE, Beck $\mathrm{J}$, et al. The Holstein Friesian lethal haplotype $5(\mathrm{HH} 5)$ results from a complete deletion of TBF1M and cholesterol deficiency (CDH) from an ERV-(LTR) insertion into the coding region of APOB. PLOS One. 2016;11:e0154602.

16. Null DJ, Huthcins JL, Bickhart DM, VanRaden PM, Cole JB. Discovery of a haplotype affecting fertility in Ayrshire dairy cattle and identification of a putative causal variant. J Dairy Sci. 2017;100:S199.

17. Guarini AR, Sargolzaei M, Brito LF, Kroezen V, Lourenco DAL, Baes CF, et al. Estimating the effect of the deleterious recessive haplotypes AH1 and $\mathrm{AH} 2$ on reproduction performance of Ayrshire cattle. J Dairy Sci. 2019:102:5315-22.

18. Cooper TA, Wiggans GR, Null DJ, Hutchison JL, Cole JB. Genomic evaluation, breed identification, and discovery of a haplotype affecting fertility for Ayrshire dairy cattle. J Dairy Sci. 2014;97:3878-82.

19. VanRaden PM, Olson KM, Null DJ, Hutchison JL. Harmful recessive effects on fertility detected by absence of homozygous haplotypes. J Dairy Sci. 2011;94:6153-61.

20. Ben Braiek M, Fabre S, Hozé C, Astruc J-M, Moreno-Romieux C. Identification of homozygous haplotypes carrying putative recessive lethal mutations that compromise fertility traits in French Lacaune dairy sheep. Genet Sel Evol. 2021;53:41.

21. Derks MFL, Megens HJ, Bosse M, Lopes MS, Harlizius B, Groenen MAM. A systematic survey to identify lethal recessive variation in highly managed pig populations. BMC Genomics. 2017;18:858.

22. Derks MFL, Megens HJ, Bosse M, Visscher J, Peeters K, Bink M, et al. A survey of functional genomic variation in domesticated chickens. Genet Sel Evol. 2018;50:17.

23. Georges $M$, Charlier C, Hayes B. Harnessing genomic information for livestock improvement. Nat Rev Genet. 2019;20:135-56. 
24. Abdalla EA, Id-Lahoucine S, Cánovas A, Casellas J, Schenkel FS, Wood $B J$, et al. Discovering lethal alleles across the turkey genome using a transmission ratio distortion approach. Anim Genet. 2020;51:876-89.

25. Hoff JL, Decker JE, Schnabel RD, Taylor JF. Candidate lethal haplotypes and causal mutations in Angus cattle. BMC Genomics. 2017;18:799.

26. Mesbah-Uddin M, Hoze C, Michot P, Barbat A, Lefebvre R, Boussaha M, et al. A missense mutation (p.Tyr452Cys) in the CAD gene compromises reproductive success in French Normande cattle. J Dairy Sci. 2019;102:6340-56.

27. Fritz S, Capitan A, Djari A, Rodriguez SC, Barbat A, Baur A, et al. Detection of haplotypes associated with prenatal death in dairy cattle and identification of deleterious mutations in GART, SHBG and SLC37A2. PLoS One. 2013;8:e65550.

28. Michot P, Fritz S, Barbat A, Boussaha M, Deloche MC, Grohs C, et al. A missense mutation in PFAS (phosphoribosylformylglycinamidine synthase) is likely causal for embryonic lethality associated with the $\mathrm{MH} 1$ haplotype in Montbeliarde dairy cattle. J Dairy Sci. 2017;100:8176-87.

29. Fritz S, Hoze C, Rebours E, Barbat A, Bizard M, Chamberlain A, et al. An initiator codon mutation in SDE2 causes recessive embryonic lethality in Holstein cattle. J Dairy Sci. 2018;101:6220-31.

30. Hozé C, Escouflaire C, Mesbah-Uddin M, Barbat A, Boussaha M, Deloche MC, et al. Short communication: A splice site mutation in CENPU is associated with recessive embryonic lethality in Holstein cattle. J Dairy Sci. 2020;103:607-12.

31. Kunz E, Rothammer S, Pausch H, Schwarzenbacher H, Seefried FR, Matiasek K, et al. Confirmation of a non-synonymous SNP in PNPLA8 as a candidate causal mutation for Weaver syndrome in Brown Swiss cattle. Genet Sel Evol. 2016;48:21.

32. Charlier C, Li W, Harland C, Littlejohn M, Coppieters W, Creagh F, et al. NGS-based reverse genetic screen for common embryonic lethal mutations compromising fertility in livestock. Genome Res. 2016;26:1333-41.

33. McClure MC, Bickhart D, Null D, VanRaden $P, X u L Y$, Wiggans $G$, et al. Bovine exome sequence analysis and targeted SNP genotyping of recessive fertility defects $\mathrm{BH} 1, \mathrm{HH} 2$, and $\mathrm{HH} 3$ reveal a putative causative mutation in SMC2 for HH3. PLoS One. 2014;9:e92769.

34. Hagger C. Estimates of genetic diversity in the brown cattle population of Switzerland obtained from pedigree information. J Anim Breed Genet. 2005;122:405-13.

35. Signer-Hasler H, Burren A, Neuditschko M, Frischknecht M, Garrick D, Stricker $C$, et al. Population structure and genomic inbreeding in nine Swiss dairy cattle populations. Genet Sel Evol. 2017;49:83.

36. National Center for Biotechnology Information. ARS-UCD1.2 Assembly. https://www.ncbi.nlm.nih.gov/assembly/GCF_002263795.1. Accessed 2021 Apr 15.

37. Rosen BD, Bickhart DM, Schnabel RD, Koren S, Elsik CG, Tseng E, et al. De novo assembly of the cattle reference genome with single-molecule sequencing. Gigascience. 2020;9:giaa021. https://doi.org/10.1093/gigas cience/giaa021

38. Sargolzaei M, Chesnais JP, Schenkel FS. A new approach for efficient genotype imputation using information from relatives. BMC Genomics. 2014; 15:478.

39. Browning SR, Browning BL. Rapid and accurate haplotype phasing and missing-data inference for whole-genome association studies by use of localized haplotype clustering. Am J Hum Genet. 2007;81:1084-97.

40. Sargolzaei M. SNP1101 User's Guide. Version 1.0. Guelph: HiggsGene Solutions Inc.; 2014

41. Wigginton JE, Cutler DJ, Abecasis GR. A note on exact tests of HardyWeinberg equilibrium. Am J Hum Genet. 2005;76:887-93.

42. Benjamini Y, Yekutieli D. The control of the false discovery rate in multiple testing under dependency. Ann Stat. 2001;29:1165-88.

43. Garrick DJ, Taylor JF, Fernando RL. Deregressing estimated breeding values and weighting information for genomic regression analyses. Genet Sel Evol. 2009;41:55

44. Yang J, Lee SH, Goddard ME, Visscher PM. GCTA: A tool for genomewide complex trait analysis. Am J Hum Genet. 2011;88:76-82.

45. VanRaden PM. Efficient methods to compute genomic predictions. J Dairy Sci. 2008;91:4414-23.

46. Turner S. qqman: an R package for visualizing GWAS results using Q-Q and manhattan plots. J Open Source Softw. 2018;3:1-2.

47. Chen S, Zhou Y, Chen Y, Gu J. fastp: an ultra-fast all-in-one FASTQ preprocessor. Bioinformatics. 2018;34:1884-90.
48. Li H, Durbin R. Fast and accurate short read alignment with BurrowsWheeler transform. Bioinformatics. 2009;25:1754-60.

49. Broad Institute. Picard Tools version 2.18.2. http://broadinstitute. github.io/picard/. 2018. Accessed 2021 Apr 15.

50. DePristo MA, Banks E, Poplin R, Garimella KV, Maguire JR, Hartl C, et al. A framework for variation discovery and genotyping using nextgeneration DNA sequencing data. Nat Genet. 2011;43:491-8.

51. Bovine Research Community. 1000 Bull Genomes Project. http:// www.1000bullgenomes.com/. Accessed 2018 Jun 15.

52. Li H, Handsaker B, Wysoker A, Fennell T, Ruan J, Homer N, et al. The sequence alignment/map format and SAMtools. Bioinformatics. 2009:25:2078-9.

53. Brentp/goleft. Github. https://github.com/brentp/goleft. Accessed 2018 Aug 20.

54. National Center for Biotechnology Information. NCBI Bos taurus Annotation Release 106. https://www.ncbi.n/m.nih.gov/genome/ annotation_euk/Bos_taurus/106/. Accessed 2018 Aug 20.

55. Cingolani P, Patel VM, Coon M, Nguyen T, Land SJ, Ruden DM, et al. Using Drosophila melanogaster as a model for genotoxic chemical mutational studies with a new program. SnpSift Front Genet. 2012;3:35.

56. Hayes BJ, Daetwyler HD. 1000 bull genomes project to map simple and complex genetic traits in cattle: applications and outcomes. Annu Rev Anim Biosci. 2019;7:89-102.

57. Purcell S, Neale B, Todd-Brown K, Thomas L, Ferreira MA, Bender D, et al. PLINK: a tool set for whole-genome association and populationbased linkage analyses. Am J Hum Genet. 2007:81:559-75.

58. Hu Y, Yan C, Hsu CH, Chen QR, Niu K, Komatsoulis GA, et al. OmicCircos: a simple-to-use R package for the circular visualization of multidimensional Omics data. Cancer Inform. 2014;13:13-20.

59. Kuhn RM, Haussler D, Kent JW. The UCSC genome browser and associated tools. Brief Bioinform. 2013;14:144-61.

60. Lander ES, Linton LM, Birren B, Nusbaum C, Zody MC, Baldwin J, et al. Initial sequencing and analysis of the human genome. Nature. 2001;409:860-921.

61. Pollard KS, Hubisz MJ, Rosenbloom KR, Siepel A. Detection of nonneutral substitution rates on mammalian phylogenies. Genome Res. 2010:20:110-21.

62. Siepel A, Bejerano G, Pedersen JS, Hinrichs AS, Hou M, Rosenbloom K, et al. Evolutionarily conserved elements in vertebrate, insect, worm, and yeast genomes. Genome Res. 2005;15:1034-50.

63. Choi Y, Sims GE, Murphy S, Miller JR, Chan AP. Predicting the functional effect of amino acid substitutions and Indels. PLoS One. 2012; 7:e46688

64. Pejaver V, Urresti J, Lugo-Martinez J, Pagel KA, Lin GN, Nam HJ, et al. Inferring the molecular and phenotypic impact of amino acid variants with MutPred2. Nat Commun. 2020;11:5918.

65. Cheong A, Lingutla R, Mager J. Expression analysis of mammalian mitochondrial ribosomal protein genes. Gene Expr Patterns. 2020;38:119147.

66. Puebla-Osorio N, Lacey DB, Alt FW, Zhu C. Early embryonic lethality due to targeted inactivation of DNA ligase III. Mol Cell Biol. 2006;26:3935-41.

67. Bowman TA, O'Keeffe KR, D'Aquila T, Yan QW, Griffin JD, Killion EA, et al. Acyl CoA synthetase 5 (ACSL5) ablation in mice increases energy expenditure and insulin sensitivity and delays fat absorption. Mol Metab. 2016:5:210-20.

68. Bayat $\mathrm{V}$, Thiffault I, Jaiswal M, Tétreault M, Donti T, Sasarman F, et al. Mutations in the mitochondrial methionyl-tRNA synthetase cause a neurodegenerative phenotype in flies and a recessive ataxia (ARSAL) in humans. PLOS Biol. 2012;10:e1001288.

69. El-Hamidi M, Leipold HW, Vestweber JGE, Saperstein G. Spinal muscular atrophy in Brown Swiss calves. Zentralbl Veterinarmed A. 1989:36:731-8.

70. Krebs S, Medugorac I, Röther S, Strässer K, Förster M. A missense mutation in the 3-ketodihydrosphingosine reductase FVT1 as candidate causal mutation for bovine spinal muscular atrophy. Proc Natl Acad Sci USA. 2007;104:6746-51.

71. Leipold HW, Blaugh B, Huston K, Edgerly CG, Hibbs CM. Weaver syndrome in Brown Swiss cattle: clinical signs and pathology. Vet Med Small Anim Clin. 1973;68:645-7. 
72. Joller S, Stettler M, Locher I, Dettwiler M, Seefried F, Meylan M, et al. Fanconi-bickel-syndrom: A novel genetic disease in Original Braunvieh. Schweiz Arch Tierheilkd. 2018;160:179-84.

73. Zhuang $Y$, Soriano $P$, Weintraub $H$. The helix-loop-helix gene E2A is required for B cell formation. Cell. 1994;79:875-84.

74. Liu W, Liu Y, Guo T, Hu C, Luo H, Zhang L, et al. TCF3, a novel positive regulator of osteogenesis, plays a crucial role in miR-17 modulating the diverse effect of canonical Wnt signaling in different microenvironments. Cell Death Dis. 2013:4:e539.

75. Narasimhan VM, Xue Y, Tyler-Smith C. Human knockout carriers: dead, diseased, healthy, or improved? Trends Mol Med. 2016;22:341-51.

76. Nosková A, Bhati M, Kadri NK, Crysnanto D, Neuenschwander S, Hofer A, et al. Characterization of a haplotype-reference panel for genotyping by low-pass sequencing in Swiss Large White pigs. BMC Genomics. 2021;22:290.

77. Ros-Freixedes R, Gonen S, Gorjanc G, Hickey JM. A method for allocating low-coverage sequencing resources by targeting haplotypes rather than individuals. Genet Sel Evol. 2017;49:78.

78. Mai N, Chrzanowska-Lightowlers ZMA, Lightowlers RN. The process of mammalian mitochondrial protein synthesis. Cell Tissue Res. 2017;367:5-20.

79. Cheong A, Archambault D, Degani R, Iverson E, Tremblay KD, Mager J. Nuclear-encoded mitochondrial ribosomal proteins are required to initiate gastrulation. Development. 2020. https://doi.org/10.1242/dev 188714.

80. Tselykh TV, Roos C, Heino TI. The mitochondrial ribosome-specific MrpL55 protein is essential in Drosophila and dynamically required during development. Exp Cell Res. 2005;307:354-66.

81. Seegers H, Beaudeau F, Fourichon C, Bareille N. Reasons for culling in French Holstein cows. Prev Vet Med. 1998;36:257-71.

82. Rilanto T, Reimus K, Orro T, Emanuelson U, Viltrop A, Mõtus K. Culling reasons and risk factors in Estonian dairy cows. BMC Vet Res. 2020;16:173.

83. Carrasco P, Jacas J, Sahún I, Muley H, Ramírez S, Puisac B, et al. Carnitine palmitoyltransferase $1 \mathrm{C}$ deficiency causes motor impairment and hypoactivity. Behav Brain Res. 2013;256:291-7.

84. Webb BD, Wheeler PG, Hagen JJ, Cohen N, Linderman MD, Diaz GA, et al. Novel, compound heterozygous, single-nucleotide variants in MARS2 associated with developmental delay, poor growth, and sensorineural hearing loss. Hum Mutat. 2015;36:587-92.

85. Simsek D, Furda A, Gao Y, Artus J, Brunet E, Hadjantonakis A-K, et al. Crucial role for DNA ligase III in mitochondria but not in Xrcc1-dependent repair. Nature. 2011;471:245-8.

86. Gao Y, Katyal S, Lee Y, Zhao J, Rehg JE, Russell HR, et al. DNA ligase III is critical for mtDNA integrity but not Xrcc1-mediated nuclear DNA repair. Nature. 2011:471:240-4

87. Murphy SM, Preble AM, Patel UK, O'Connell KL, Dias DP, Moritz M, et al. GCP5 and GCP6: two new members of the human gamma-tubulin complex. Mol Biol Cell. 2001;12:3340-52.

88. Izumi N, Fumoto K, Izumi S, Kikuchi A. GSK-3 $\beta$ regulates proper mitotic spindle formation in cooperation with a component of the $\gamma$-tubulin ring complex, GCP5. J Biol Chem. 2008;283:12981-91.

89. Farache $D$, Emorine $L$, Haren L, Merdes A. Assembly and regulation of -tubulin complexes. Open Biol. 2018;8:170266.

90. Maver A, Čuturilo G, Kovanda A, Miletić A, Peterlin B. Rare missense TUBGCP5 gene variant in a patient with primary microcephaly. Eur J Med Genet. 2019;62:103598.

91. Cheng JB, Jacquemin E, Gerhardt M, Nazer H, Cresteil D, Heubi JE, et al. Molecular genetics of $3 \beta$-hydroxy- $\triangle 5-C 27$-steroid oxidoreductase deficiency in 16 patients with loss of bile acid synthesis and liver disease. J Clin Endocrinol Metab. 2003;88:1833-41.

92. Shea HC, Head DD, Setchell KDR, Russell DW. Analysis of HSD3B7 knock out mice reveals that a $3 a$-hydroxyl stereochemistry is required for bile acid function. Proc Natl Acad Sci USA. 2007;104:11526-33.

93. Bhati M, Kadri NK, Crysnanto D, Pausch H. Assessing genomic diversity and signatures of selection in Original Braunvieh cattle using wholegenome sequencing data. BMC Genomics. 2020;21:27.

94. Millay DP, O'Rourke JR, Sutherland LB, Bezprozvannaya S, Shelton JM, Bassel-Duby R, et al. Myomaker is a membrane activator of myoblast fusion and muscle formation. Nature. 2013;499:301-5.
95. Derks MFL, Lopes MS, Bosse M, Madsen O, Dibbits B, Harlizius B, et al. Balancing selection on a recessive lethal deletion with pleiotropic effects on two neighboring genes in the porcine genome. PLoS Genet. 2018;14:e1007661.

96. Kadri NK, Sahana G, Charlier C, Iso-Touru T, Guldbrandtsen B, Karim L, et al. A 660-Kb deletion with antagonistic effects on fertility and milk production segregates at high frequency in Nordic Red cattle: Additional evidence for the common occurrence of balancing selection in livestock. PLoS Genet. 2014;10:e1004049.

97. Crysnanto D, Leonard AS, Fang Z-H, Pausch H. Novel functional sequences uncovered through a bovine multi-assembly graph. Proc Natl Acad Sci USA. 2021;118:e2101056118.

98. Posey JE. Genome sequencing and implications for rare disorders. Orphanet J Rare Dis. 2019;14:153.

99. Bocher O, Génin E. Rare variant association testing in the non-coding genome. Hum Genet. 2020;139:1345-62.

100. Menzi F, Besuchet-Schmutz N, Fragnière M, Hofstetter $S$, Jagannathan $V$, Mock T, et al. A transposable element insertion in APOB causes cholesterol deficiency in Holstein cattle. Anim Genet. 2016;47:253-7.

101. Häfliger IM, Marchionatti E, Stengård M, Wolf-Hofstetter S, Paris JM, Jacinto JG, et al. CNGB3 missense variant causes recessive achromatopsia in Original Braunvieh cattle. Int J Mol Sci. 2021;22:12440.

\section{Publisher's Note}

Springer Nature remains neutral with regard to jurisdictional claims in published maps and institutional affiliations.

Ready to submit your research? Choose BMC and benefit from:

- fast, convenient online submission

- thorough peer review by experienced researchers in your field

- rapid publication on acceptance

- support for research data, including large and complex data types

- gold Open Access which fosters wider collaboration and increased citations

- maximum visibility for your research: over 100M website views per year

At BMC, research is always in progress.

Learn more biomedcentral.com/submissions 